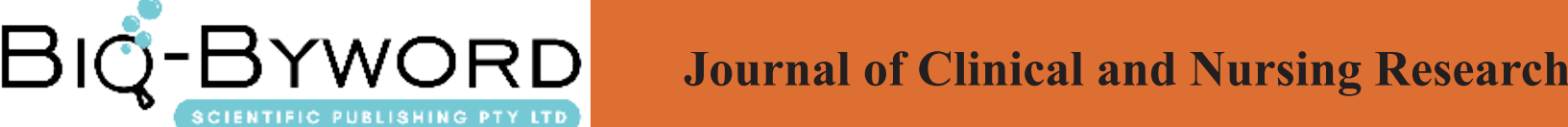

\section{Effect of Recombinant Human Lactoferrin on Allergic Rhinitis in Mice Nasal Drip and Its Mechanism}

FANG Rui-ping, GAO Hong-yan*, WANG Xiao-li, YANG Xiao-ping, LI Li

\section{$X i$ 'an Children's Hospital ENT Shaanxi Xi'an}

\section{ARTICLE INFO}

Article history:

Published online: 15th July, 2017

Key words:

Allergic rhinitis

lactoferrin; eosinophils

cytokines

T cells

\section{ABSTRACT}

Abstract: Objective: To study the effect of recombinant human lactoferrin on allergic rhinitis in mice nasal drip and explore its possible molecular mechanism. Methods: The animal model of allergic rhinitis in BALB / c mice was established by ovalbumin. The eosinophils, goblet cells and mast cell infiltration of nasal mucosa were analyzed by hematoxylin-eosin, happening. The transcription factors and cytokines and expression levels of lactoferrin gene and protein of $\mathrm{T}$ lymphocyte subsets in nasal cavity were detected by fluorescence quantitative RT-PCR and enzyme-linked immunosorbent assay. Spearman was used to analyzing the relationship between lactoferrin expression levels and eosinophils in the nasal cavity. Results: The number of eosinophils, goblet cells and mast cells, Th2, Th17, Treg gene and protein expression levels in nasopharyngeal neonatal mice were significantly higher than those in normal mice $(\mathrm{P}<0.05)(\mathrm{P}<0.05)$. The levels of recombinant human lactoferrin were significantly decreased before and after ovariectomy $(\mathrm{P}<0.05)$. Th1-related gene and protein expression levels were not significantly different between normal mice and model mice, but were significantly upregulated after recombinant human lactoferrin $(\mathrm{P}<0.05)$. Compared with normal mice, the expression level of endogenous lactoferrin was significantly down-regulated in nasal cavity of allergic rhinitis mice $(\mathrm{P}<0.05)$, but was significantly up-regulated after treatment with recombinant human lactoferrin $(\mathrm{P}<0.05)$. Spearman correlation analysis showed that the expression level of lactoferrin was negatively correlated with the number of eo-

*Corresponding author

Gao Hongyan. Xi'an Children's Hospital ENT

Shaanxi Xi'an 
sinophils $(r=-0.920, P<0.05)$. Conclusion: Endogenous lactoferrin is down-regulated in the nasal cavity of allergic rhinitis mice. The lack of protein expression may be related to the occurrence and development of allergic rhinitis. Exogenous lactoferrin inhibits inflammation of allergic rhinitis in mice, and its

\section{Introduction}

Allergic rhinitis is a major pathological feature of T cell subsets, eosinophils and mast cell infiltration, among which $\mathrm{T}$ lymphocyte subsets play a central role in the immune response. CD4 + T cell subsets can be divided into type I helper T cells (Th1), Th2, and regulatory T cells (Treg) according to their phenotype and functional properties. These effects of $T$ cell subsets regulate allergic diseases mainly by secreting specific cytokines such as IFN- $\gamma$, IL-5, IL-17, IL-10 and TGF- $\beta 1$, and by secreting other non-specific Cytokines such as TNF- $\alpha$ ${ }^{[1-2]}$. A study abroad found that serum levels of lactoferrin in patients with allergic rhinitis were lower than those in normal subjects and were positively correlated with the severity of the disease, suggesting that insufficient expression of lactoferrin in peripheral blood may be involved in the development of allergic rhinitis and Development ${ }^{[3]}$. By giving exogenous lactoferrin to supplement the endogenous lactoferrin secretion, can reverse the process of allergic rhinitis, at home and abroad have not been related research and reports. In view of the anti-inflammatory and immunomodulatory properties of lactoferrin and the ability to regulate the Th1 and Th2 immune responses under physiological and pathological conditions, this has led us to investigate the effects of lactoferrin in the allergic rhinitis model may regulate the role and explore its potential effect mechanism.

\section{Materials and methods}

\subsection{Materials}

Experimental animals and experimental groups: 5-6 weeks of age SPF grade BALB / c mice (20-27 g) were purchased from our laboratory animal center. Forty male mice were randomly divided into normal control group, model group, prevention group and treatment group, 10 mice in each group. Feeding conditions: 5 per cage, room temperature 20-28 degrees, relative humidity $40 \%-80 \%$, noise $<60 \mathrm{db}$. All animals are tested in accordance with the relevant principles of the Committee on Animal Management and Use in our hospital.

The main reagents: ovalbumin (OVA) US Sigma; hematoxylin and yinhong dye Shanghai Biyun days Bio- technology Co., Ltd.; Trizol United States Invitrogen; DEPC Wuhan Google Biotechnology Co., Ltd.; Primer Shanghai Biology Limited Engineering Company Design and synthesis; Rever Tra Ace RT Kit Japan TOYOBO; Rever Tra Ace qPCR Kit Japan TOYOBO; SPSS Statistics 19.0 United States IBM; recombinant human lactoferrin in the United States, the United States, the United States, the United States and the United States, PeproTech company; mast cell kit Fuzhou Mai new biotechnology company.

\subsection{Methods}

1.2.1 Establishment of mouse allergic rhinitis model. 40 male mice were randomly divided into normal control group (group A) and model group (group B), and 24 hours before lactoferrin challenge group (group C) , 6 hours after lactoferrin challenge were given to the treatment group (group D), 10 mice in each group. According to the literature method, ovalbumin (OVA) was used to sensitize and stimulate allergen, and the model of allergic rhinitis was established ${ }^{[4]}$.

1.2.2 Nasal irrigation. Control group and model group randomly selected 5 mice. Mice were treated with $1 \%$ pentobarbital sodium $50 \mathrm{mg} / \mathrm{kg}$ intraperitoneally. The neck was tracheotomy after deep anesthesia and inserted into the posterior nostrils from the trachea opening in the direction of the anterior nostril with a $1 \mathrm{ml}$ syringe (rounded) Each nostril was gently poured into $1 \mathrm{ml}$ of sterile saline and the laceration was collected from the previous nostrils. The collected nasal lavage fluid was then centrifuged at $12000 \mathrm{rpm}$ for 10 minutes and the resulting supernatant was stored in a refrigerator at -20 ${ }^{\circ} \mathrm{C}$ for enzyme-linked immunosorbent assay (ELISA) to determine the concentration of lactoferrin.

1.2.3 Nasal Lavage Fluid Cytokine Assay Enzyme-linked immunosorbent assay kit. For the determination of lactoferrin concentration in nasal lavage fluid was purchased from Wuhan Bude Biotechnology Co., Ltd., China. ELISA kit detection sensitivity is less than $2 \mathrm{pg} / \mathrm{ml}$. Operation method in strict accordance with the instructions provided by the kit to carry out this experiment using double antibody sandwich method.

1.2.4 Tissue treatment and HE staining. Nasal mu- 
Table 1 Number of eosinophils in each group (/ mm2)

\begin{tabular}{ccc}
\hline Group & $\mathrm{N}$ & Eosinophils \\
\hline Control group & 5 & $5.29 \pm 0.50$ \\
Model group & 5 & $92.35 \pm 8.24^{*}$ \\
Prevention group & 5 & $35.78 \pm 3.29^{* \#}$ \\
Treatment group & 5 & $54.68 \pm 5.21^{* \# \mathrm{~S}}$ \\
\hline
\end{tabular}

Compared with the control group, ${ }^{\star} \mathrm{p}<0.05$; compared with the model group, $\# \mathrm{p}<0.05$; compared with the prevention group, $\$ \mathrm{p}<0.05$

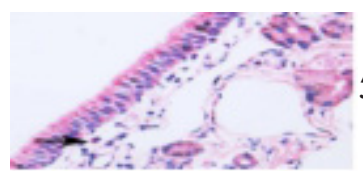

Control group

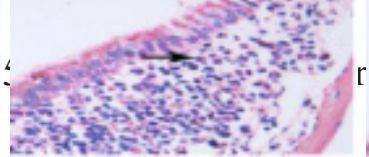

Model group

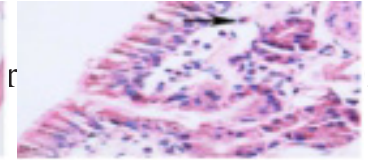

Prevention group

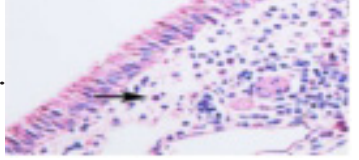

Treatment group

Figure 1 mouse nasal mucosa HE staining results $(\times 400)$

cosal histopathological changes were hematoxylin-eosin staining showed eosinophils, high iodine sparse staining showed goblet cells and mucus cells, mast cell staining showed infiltration of mast cells. $\left(\mathrm{N} / \mathrm{mm}^{2}\right)$ in the lamina propria of the nasal mucosa per square millimeter, and the mean values of the four high power fields were observed in each mouse. The goblet cells are expressed as the number of goblet cells per millimeter of basement membrane $(\mathrm{N} / \mathrm{mm})$, and mast cells are expressed as the number of mast cells per high power field ( $\mathrm{N} / \mathrm{HP})$.

\subsubsection{RNA extraction and real-time quantitative RT-}

PCR. Under the dissecting microscope, the mouse nasal mucosa was stripped with micro-tooth tweezers and immediately placed in a $5 \mathrm{ml}$ homogenizer until there was no obvious tissue mass in the naked eye. According to the literature method, take $2 \mathrm{ul}$ RNA directly in the spectrophotometer on the determination of RNA concentration, and all the samples to be measured all the RNA concentration adjusted to $0.5 \mathrm{ug} / \mathrm{ul},-70$ degrees preservation. Detection of the expression level of the target gene, according to previously reported method ${ }^{[4]}$. Analysis and calculation of the relative expression data of the target gene was performed automatically using the 2- $\Delta \Delta \mathrm{CT}$ method inherent in the ABI 7500 sequence detection system software (Applied Biosystems).

\subsection{Statistical analysis}

All data were expressed as $\overline{\mathrm{x}}+\mathrm{s}$, and the results of each group were first tested with nonparametric Kruskal-Wallis test. If there were statistical differences, then Mann-Whitney U was used for comparison. Spearman correlation analysis of the expression of lactoferrin in the nasal cavity and the correlation of eosinophils. Statistical analysis was performed using IBM SPSS Statistics 19.0 (IBM, USA) software, with $\mathrm{P}<0.05$ as a statistically significant test.

\section{Results}

The effect of nasal recombinant lactoferrin on the number of eosinophils infiltrated in nasal mucosa

As shown in Figure 1 and Table 1, HE staining confirmed that the model group had significant eosinophils in the nasal mucosa. The cells are mainly located in the lamina propria layer, a few distributed between the epithelium, cytoplasm dyed red. In addition also visible lymphocytes and monocytes and other inflammatory cells. In the normal control group, only a small number of mucosal lamina propria scattered in the distribution of eosinophils. Compared with the normal control group, the number of eosinophils in the nasal mucosa of the model group was significantly increased, accompanied by a relatively large number of lymphocytes and mononuclear cells, and a few epithelial lesions ( $\mathrm{P}$ $<0.05$ ). The eosinophils in the nasal mucosa of the mice were significantly decreased after the recombinant lactoferrin was injected into the nasal cavity, and the effect of the instillation of the recombinant lactoferrin was more obvious $(\mathrm{P}<0.05)$.

2.2 Nasal recombinant lactoferrin reduces the number of goblet cells in the nasal mucosa

As shown in Figure 2 and Table 2, the cytoplasm of goblet cells is stained purple. In the normal control 
Table 2 Four groups of mice nasal mucosa number of goblet cells $\left(/ \mathrm{mm}^{2}\right)$

\begin{tabular}{ccc}
\hline Group & $\mathrm{N}$ & Goblet cells \\
\hline Control group & 5 & $7.08 \pm 0.68$ \\
Model group & 5 & $26.84 \pm 2.35^{*}$ \\
Prevention group & 5 & $12.89 \pm 1.34^{* \#}$ \\
Treatment group & 5 & $19.08 \pm 1.86^{* \# \mathrm{~S}}$ \\
\hline
\end{tabular}

Compared with the control group, ${ }^{\star} \mathrm{p}<0.05$; compared with the model group, $\# \mathrm{p}<0.05$; compared with the prevention group, $\$ \mathrm{p}<0.05$

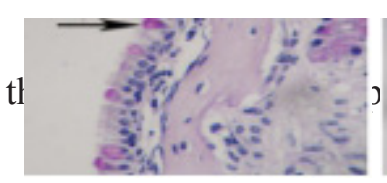

Control group

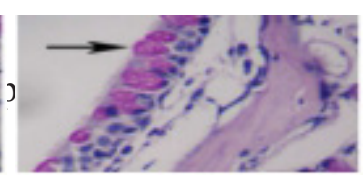

Model group

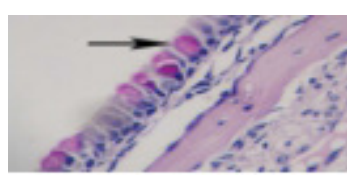

Prevention group

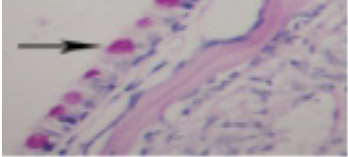

Treatment group

Figure 2 four groups of mice nasal mucosa PAS staining results $(\times 400)$

group, only a small number of mucosal epithelium was scattered in the distributed goblet cells. Compared with the normal control group, the number of goblet cells in the nasal mucosa of the model group was significantly higher than that of the normal control group, and the cytoplasm was hyperplastic hypertrophy and some goblet cells showed typical goblet $(\mathrm{P}<0.05)$. After intragastric administration of recombinant lactoferrin, the goblet cells in the nasal mucosa were significantly decreased, and the effect of the instillation of the recombinant lactoferrin was more obvious $(\mathrm{P}<0.05)$.

\subsection{Nasal Recombinant Lactoferrin reduces the number}

of granulocytes infiltrated in the nasal mucosa

As shown in Figure 3, Table 3, mast cell cytoplasm dyed dark purple. In the normal control group, only a small number of mucosal epithelial cells scattered in the distribution of mast cells. Compared with the normal control group, the mast cells in the nasal mucosa of the model group were significantly increased, the number of granules in the cytoplasm was significantly increased, and the mast cells were mostly distributed in the olfactory nerve fibers and the blood vessels $(\mathrm{P}<$ 0.05). The mast cells in the nasal mucosa of the mice were significantly decreased after the incorporation of

Table 3 four groups of mice nasal mucosa hypertrophy granulocyte number $\left(/ \mathrm{mm}^{2}\right)$

\begin{tabular}{ccc}
\hline Group & $\mathrm{N}$ & Hypertrophy granulocytes \\
\hline Control group & 5 & $3.96 \pm 0.35$ \\
Model group & 5 & $26.79 \pm 2.15^{*}$ \\
Prevention group & 5 & $5.05 \pm 0.48^{* \#}$ \\
Treatment group & 5 & $10.59 \pm 1.02^{* \# \$}$ \\
\hline
\end{tabular}

Compared with the control group, ${ }^{\star} \mathrm{p}<0.05$; compared with the model group, $\# \mathrm{p}<0.05$; compared with the prevention group, $\$ \mathrm{p}<0.05$

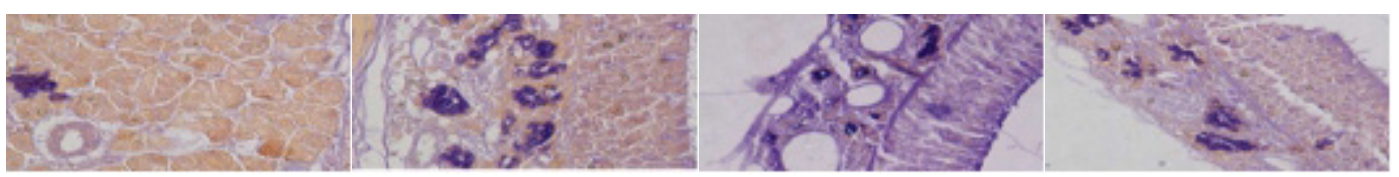
Control group
Model group
Prevention group
Treatment group

Figure 3 four groups of mice nasal mucosa mast cell staining results $(\times 400)$ 
Table 4 The expression levels of cytokines IFN- $\gamma$, IL-5, IL-10, IL-17, TGF- $\beta 1$ and LF in parenchymal lavage fluid of four groups ( $\mathrm{pg} / \mathrm{ml}$ )

\begin{tabular}{|c|c|c|c|c|c|c|}
\hline Group & IFN- $\gamma$ & IL-5 & IL-10 & IL-17 & TGF- $\beta 1$ & LF \\
\hline $\begin{array}{c}\text { Control } \\
\text { group }\end{array}$ & $16.79 \pm 1.59$ & $7.25 \pm 0.70$ & $38.79 \pm 3.69$ & $6.28 \pm 0.60$ & $66.84 \pm 6.36$ & $46.37 \pm 4.52$ \\
\hline Model group & $27.05 \pm 2.52^{*}$ & $31.54 \pm 3.28^{*}$ & $84.96 \pm 8.22^{*}$ & $18.39 \pm 1.65^{*}$ & & $32.59 \pm 3.08^{*}$ \\
\hline $\begin{array}{c}\text { Prevention } \\
\text { group }\end{array}$ & $40.28 \pm 3.86^{* \#}$ & $9.56 \pm 0.94^{* \#}$ & $42.56 \pm 4.08^{* \#}$ & $7.25 \pm 0.71^{* \#}$ & $78.26 \pm 7.51^{* \#}$ & $72.12 \pm 7.03^{* \#}$ \\
\hline $\begin{array}{c}\text { Treatment } \\
\text { group }\end{array}$ & & & & & & \\
\hline
\end{tabular}

Compared with the control group, ${ }^{*} \mathrm{p}<0.05$; compared with the model group, ${ }^{*} \mathrm{p}<0.05$; compared with the prevention group, ${ }^{\$} \mathrm{p}<0.05$

recombinant lactoferrin, and the effect of the recombinant lactoferrin was more obvious $(\mathrm{P}<0.05)$.

2.4 Effect of nasal recombinant lactoferrin on different cytokines in the nasal cavity

As shown in Table 4, the level of IFN- $\gamma$ in the nasal lavage fluid was significantly upregulated in the model group compared with the normal control group, and further increased after nasal recuperated lactoferrin treatment, and the effect was more pronounced before the challenge $<0.05$ ). Compared with the normal control group, the levels of IL-5, IL-10, IL-17 and TGF- $\beta 1$ in nasal lavage fluid were significantly up-regulated in the model group, but after treatment with nasal recombinant lactoferrin $(\mathrm{P}<0.05)$, and the inhibitory effect was more obvious $(\mathrm{P}<0.05)$. In contrast to the above factors, the expression level of lactoferrin in the nasal lavage fluid was significantly lower in the model group than in the normal control group, and the expression of lactoferrin was up-regulated after nasal recombination, and the effect of pre-excitation administration was more pronounced $\mathrm{P}<0.05$ ).

2.5 Different Effects of Nasal Recombinant Lactoferrin on T Cell Cytokines and Transcription Factors in Nasal Mucosa

As shown in Table 5, Th1-associated cytokines and transcription factors (IFN- $\gamma$ and T-bet) in nasal mucosa were similar in normal controls and model groups, but were further treated with nasal recombinant lactoferrin $(\mathrm{P}<0.05)$, and the effect was more obvious ( $\mathrm{P}$ $<0.05)$. Th2-related cytokines and transcription factors (IL-5 and GATA-3), Th17-related cytokines and transcription factors (IL-17 and ROR-c), Treg-associated cytokines and transcription (IL-10, Foxp3 and

Table 5 Mouse nasal mucosa T cell cytokines and transcription factor gene expres-

\begin{tabular}{ccccccc}
\hline Group & IFN- $\gamma$ & IL-5 & IL-10 & IL-17 & TGF- $\beta 1$ & LF \\
\hline $\begin{array}{c}\text { Control } \\
\text { group }\end{array}$ & $16.79 \pm 1.59$ & $7.25 \pm 0.70$ & $38.79 \pm 3.69$ & $6.28 \pm 0.60$ & $66.84 \pm 6.36$ & $46.37 \pm 4.52$ \\
Model group & $27.05 \pm 2.52^{*}$ & $31.54 \pm 3.28^{*}$ & $84.96 \pm 8.22^{*}$ & $18.39 \pm 1.65^{*}$ & & $32.59 \pm 3.08^{*}$ \\
$\begin{array}{c}\text { Prevention } \\
\text { group }\end{array}$ & $40.28 \pm 3.86^{* \#}$ & $9.56 \pm 0.94^{* \#}$ & $42.56 \pm 4.08^{* \#}$ & $7.25 \pm 0.71^{* \#}$ & $78.26 \pm 7.51^{* \#}$ & $72.12 \pm 7.03^{* \#}$ \\
$\begin{array}{c}\text { Treatment } \\
\text { group }\end{array}$ & & & & & & \\
\hline
\end{tabular}

Compared with the control group, ${ }^{*} \mathrm{p}<0.05$; compared with the model group, ${ }^{\sharp} \mathrm{p}<0.05$; compared with the prevention group, ${ }^{\$} \mathrm{p}<0.05$ 
TGF- $\beta 1$ ) were significantly up-regulated in the model group, but decreased by nasal recombinant lactoferrin $(\mathrm{P}<0.05)$. Compared with the normal control group, the expression of lactoferrin in nasal nasal mucosa was significantly down-regulated in the model group, and the expression of lactoferrin was up-regulated after nasal recombination, and the effect of pre-excitation was more obvious $(\mathrm{P}<0.05)$.

\subsection{Correlation analysis}

Spearman Correlation Analysis of the Expression of Transcription Factors in Eosinophils and Different $\mathrm{T}$ Cell Subsets in Mice 's Nasal Mucosa. There was a significant positive correlation between eosinophils and Th2 transcription factor GATA-3 expression in nasal mucosa of mice $(\mathrm{r} 1=0.947, \mathrm{P}<0.05)$. And there was a significant positive correlation between the expression level of Th17 transcription factor ROR-c and rOR-c $(\mathrm{R} 2=0.935, \mathrm{P}<0.05)$. The expression of Foxp3 was positively correlated with the expression of Treg transcription factor Foxp3(R4 = 0.683, P $<0.05)$. And the expression level of Th1 transcription factor T-bet was not correlated $(\mathrm{r} 3=0.084, \mathrm{P}>0.05)$.

\section{Discussion}

Recombinant lactoferrin can bind to the lactoferrin receptor on the surface of the sensitized lymphocytes in order to inhibit the activation of sensitive lymphocytes and inflammatory factors such as IL-5 and IL-17 in the nasal cavity before ovalbumin excitation of the release, thus further reducing the degree of inflammation. In contrast, when recombinant lactoferrin is administered to the nasal cavity after ovalbumin excitation, activated Th2 and Th17 cells release a large number of inflammatory factors to promote inflammatory cascade, but lactoferrin has no inhibitory effect on these released inflammatory mediators The In addition, the respiratory epithelium also exists lactoferrin receptor, can inhibit the metal oxide caused by airway oxidative damage. Lactoferrin can regulate the nasal mucosal immune response through activation of lymphocytes and lactoferrin receptors on airway epithelial cells, but this needs to be further confirmed by detecting the expression of lactoferrin receptors on the nasal mucosa of mice.

Allergic rhinitis is an adaptive immune response disease. CD4 $+\mathrm{T}$ cells play a central role in the immune response. CD4 $+\mathrm{T}$ cells can be classified into type I helper T cells (Th1), Th2, and regulatory T cells (Treg) according to their phenotypic and functional properties. Previous studies have generally suggested that allergic rhinitis is Th1 and Th2 imbalance and Th2-based aller- gic diseases ${ }^{[5-6]}$. Th1 mainly secrete IFN- $\gamma$-mediated cellular immune response, and Th2 mainly through the secretion of IL-4, IL-5, and IL-13 and other cytokines mediated humoral immune response, and in vivo and in vitro studies have confirmed Th1 and Th2 cells in functionally suppress each other ${ }^{[7-9]}$. Our current study found that Th1 (IFN- $\gamma$ and T-bet) gene expression was slightly upregulated in the model group compared with the normal control group, but there was no significant difference, consistent with previous studies.

Th2 plays a central role in promoting allergic inflammation, which involves inducing $\mathrm{B}$ cells to produce specific IgE, mast cells and eosinophils to differentiate into peripheral blood and infiltrate peripheral tissues. Th2 mainly through the secretion of different cytokines IL-4, IL-5 and IL-13 and so on to mediate the immune response. IL-4 mainly regulates the transformation of immunoglobulins, promotes B cells to produce specific $\mathrm{IgE}$, and plays a role in early inflammation. IL-13 mainly regulates glandular hyperplasia and hypertrophy, promoting mucus secretion. IL-5 plays an important role in late inflammation. Unlike eosinophils, mast cells play a major role in allergic inflammatory phase. Mast cells, once activated, will release a variety of inflammatory mediators, including histamine, leukotriene, prostaglandin D2, promote vascular permeability, nasal discharge increased ${ }^{[10]}$. However, recent studies have found that IL-17 also promotes the development and development of allergic diseases and is closely related to glucocorticoid-resistant hormones. The expression level of IL-17 in local mucosa of patients with inflammatory diseases such as refractory allergic rhinitis and sinusitis was significantly higher than that of other patients with easy treatment ${ }^{[11]}$. Similar to previous studies, our current study found that the number of eosinophils and mast cells in mice with allergic rhinitis nasal mucosa were significantly increased, and Th2 and Th17-related cytokine expression was positively correlated. After treatment with recombinant lactoferrin, eosinophils were significantly reduced. Decreased eosinophils may be associated with downregulation of IL-5 and IL-17 cytokine expression ${ }^{[12]}$. Our study also showed that IL17 and TGF- $\beta 1$ gene expression was significantly increased in allergic rhinitis mice compared with normal mice. However, the relationship between Th17 and lactoferrin has not been previously studied and reported. Interestingly, we first found that lactoferrin significantly reduced the expression of IL-17 and ROR-C genes, but the specific mechanism of lactoferrin down-regulation of IL-17 is not clear and may be mediated by cleavage with Th17 cells Ferritin receptors to achieve, but this requires further study to confirm. 
Composed of CD4 + T cell subsets of one of the regulatory $\mathrm{T}$ cells (Treg), expression of a specific transcription factor Foxp3, through the secretion of inhibitory cytokines IL-10 or TGF- $\beta 1$ regulation of mucosal local Th1, Th2 and Th17 function in the prevention of immune activation and down-regulation of systemic and tissue-specific autoimmune diseases and inflammation Lesions and other mucosal lesions play a vital role. Treg can be divided into two forms of spontaneous and induced occurrence, the former in the thymus formation, and the latter in the peripheral tissue in the IL-10 or TGF- $\beta 1$ stimulation induced generation ${ }^{[13]}$. Although initially that IL-10 is mainly secreted by Th2, it is possible to inhibit the differentiation and development of Th1. However, subsequent studies have found that IL10 and IL-5, IL-13 and other Th2 cytokines function significantly different. The most important function of IL-10 is to induce the formation of regulatory $\mathrm{T}$ cells, thereby inhibiting inflammation and immune responses [13-14]. More importantly, allergic rhinitis patients with peripheral blood loss is induced by the formation of Treg, rather than the naturally occurring Treg ${ }^{[14]}$. In the current study, we found that IL-10 and Foxp3 gene expression was significantly upregulated in allergic rhinitis mice, but was significantly downregulated after recombinant lactoferrin therapy, suggesting that lactoferrin can inhibit regulation in vivo T cells. Our findings are consistent with previous in vitro experimental structures, ie, regulatory $\mathrm{T}$ cells are very sensitive to lactoferrin, high concentrations of lactoferrin inhibit the expression of regulatory $\mathrm{T}$ cells, whereas low concentrations of lactoferrin do not inhibit regulatory $\mathrm{T}$ cells The expression ${ }^{[10]}$. Downregulated levels of IL-10 may be associated with suppressed Th2 and Treg functions, as they are important sources of IL-10. In addition, we further found that the number of eosinophils in the nasal mucosa of mice with allergic rhinitis was positively correlated with the expression of Treg, suggesting that locally increased Treg of inflammation contributes to the reduction of inflammatory responses.

Dendritic cells (DCs) play an important role in the induction of $\mathrm{T}$ cell immune responses and are the most important antigen presenting cells (APC) that initiate the initial immune response. But dendritic cells are capable of presenting antigens, depending on their activation and maturation status. And lactoferrin can induce the maturation of MoDCs phenotype and function from mature mononuclear cells. Because lactogen-derived cells play an important role in inducing immune response, lactoferrin can promote the migration, recruitment and maturation of antigen-presenting cells, and inhibits regulatory $\mathrm{T}$ cells, suggesting that lactoferrin can enhance antigen-specific immune responses. In- deed, mice administered both lactoferrin and antigen ovalbumin can enhance mouse specific immune response, mainly produce antigen-specific IFN- $\gamma$, while down-regulating IL-4 and IL-10 expression ${ }^{[10]}$.

In addition, we further investigated the effect of recombinant lactoferrin (exogenous recombinant lactoferrin) on the expression of nasal lactoferrin (endogenous recombinant lactoferrin) in allergic rhinitis mice. Lactoferrin is present in two forms, namely secretory (sLF, in vivo) and intracellular (DeltaLF). Gene chip studies suggest that both forms of lactoferrin have different signaling pathways in immune regulation, cell growth and differentiation, suggesting that their function is different [15]. It is interesting to note that our current study found that the expression of both forms of lactoferrin (in the medial and mucous membranes) in allergic rhinitis was significantly lower than that in normal mice Lactoferrin treatment was significantly improved. This suggests that exogenous lactoferrin can induce endogenous lactoferrin expression, but its mechanism needs to be further studied.

In summary, studies have shown that allergic rhinitis is Th2 cells and Th17 cells mixed with the characteristics of the immune response, accompanied by the relative lack of Th1. Nasal externally given exogenous lactoferrin attenuates nasal inflammation in allergic rhinitis mice. Exogenous lactoferrin not only up-regulated the expression of endogenous lactoferrin, but also localized T-cell immune response in nasal mucosa of allergic rhinitis from Th2 and Th17 to Th1 cells. Due to its inhibitory properties, lactoferrin may be used as a new strategy for the prevention and treatment of allergic rhinitis in the future.

\section{References}

[1] Quan SH, Zhang YL, Han DH, et al. Contribution of Interleukin 17A to the Development and Regulation of Allergic Inflammation in a Murine Allergic Rhinitis Model [J]. Ann Allergy Asthma Immunol, 2012, 108 (5): $342-350$.

[2] Mo JH, Kang EK, Quan SH, et al. Anti-Tumor Necrosis Factor-Alpha Treatment Allergen Responses in an Allergic Rhinitis Mouse Model [J]. Allergy, 2011, 66 (2): 279-286.

[3] Choi GS, Shin SY, Kim JH, et al. Serum lactoferrin level as a serologic biomarker for allergic rhinitis [J]. Clin Exp Allergy, 2010, 40 (3): 403-410.

[4] Mo JH, Kang EK, Quan SH, et al. Anti-tumor Necrosis Factor-Alpha Treatment Allergen Responses in an Allergic Rhinitis Mouse Model [J]. Allergy, 2011, 66 
(2): 279-286.

[5] Lou Hongfei, Fu Yujing, Wang Chengsuo, et al. Umbilical Cord Th1 / Th2 Cell Imbalance and Maternal Allergic Rhinitis and Infants with Atopic Dermatitis $[\mathrm{J}]$. Chinese Journal of Otorhinolaryngology Head and Neck Surgery, 2014, 5): 390-394.

[6] Liu Weiwei, Wang Hongqin, Li Jian et al. Effects of Exogenous IL-10 On Expression of Th Cells and Th17 Cells in Allergic Rhinitis Rats [J]. Chines Journal of Microbiology and Immunology, 2014, (11): 839-843

[7] Xiao Erbin, Zhao Baocheng, Zhang Chi, et al. Adipose-Derived Mesenchymal Stem Cells Can Regulate the Immune Status of Allergic Rhinitis T Cells [J]. Chinese Journal of Tissue Engineering, 2016, 20 (10): 1373-1381.

[8] Li Linge, Feng Juan, Hu Bin et al. Experimental Study on the Construction of Experimental Allergic Rhinitis Model Mice and Th1 / Th2 Imbalance [J]. Chinese Journal of Tissue Engineering, 2015, (40): 65156519 The

[9] Lu Y, Sjostrand M, Malmhall C, et al. New Production of Eosinophils and the Corresponding TH1 / TH2 Balance in the Lungs after Allergen Exposure in BALB / $\mathrm{c}$ and C57BL / 6 Mice [J] Scand J Immunol, 2010, 71 (3): 176-185.
[10] Siracusa MC, Kim BS, Spergel JM, et al. Basophils and Allergic Inflammation [J]. J Allergy Clin Immunol, 2013, 132 (4): 789-801; quiz 788.

[11] Quan SH, Zhang YL, Han DH, et al. Contribution of Interleukin 17A to the Development and Regulation of Allergic Inflammation in a Murine Allergic Rhinitis Model [J]. Ann Allergy Asthma Immunol, 2012, 108 (5): $342-350$.

[12] Ouyang YH, Miyata M, Hatsushika K, et al. TGF- $\beta$ SignalingMay Play a Role in the Development of Goblet Cell Hyperplasia in a Mouse Model of Allergic Rhinitis [J]. Allergology International, 2010, 59 (3): 1-7.

[13] Josefowicz SZ, Niec RE, Kim Hy, et al. Extrathymically Generated Regulatory T Cells Control Mucosal TH2 Inflammation [J]. Nature, 2012, 482 (7385): 395399.

[14] Han D, Wang C, Lou W, et al. Allergen-Specific IL-10-Secreting Type IT Regulatory Cells, But Not CD4 (+) CD25 (+) Foxp3 (+) T Cells, of Patients with Persistent Allergic Rhinitis [J]. Clin Immunol, 2010, 136 (2): 292-301.

[15] Kim B, Kang S, Kim SJ. Genome-Wide Pathway Analysis Reveals Different Signaling Pathways Between Secreted Lactoferrin and Intracellular Delta-Lactoferrin [J]. PLoS One, 2013, 8(1): e55338. 